\title{
DIE BEWIRTUNG DES VOLKES: ÖFFENTLICHE SPEISUNGEN IN DER RÖMISCHEN KAISERZEIT
}

\author{
Eftychia Stavrianopoulou
}

\section{Einführung}

Einem Diktum des französischen Anthropologen Claude LÉVISTRAUSS zufolge sind Opfertiere „good to eat" und ,good to think“. Über Feste, Opfer und Bewirtungen nachzudenken, kann in der Tat einen Ansatzpunkt bilden, um dem sozialen und politischen Leben der antiken Gemeinden näher zu kommen. An solchen - wohl gemerkt gelungenen - Versuchen mangelt es nicht: Pauline SCHMITT PANTEL hat dies bereits in ihrem Buch La cité au banquet hinsichtlich der Bedeutung des gemeinsamen Festmahls in der klassischen und hellenistischen Epoche im griechischen Kulturraum dargelegt. ${ }^{2}$ Für das römische Bankett gibt es eine Reihe von Studien aus jüngerer Zeit, die das rege Interesse an der Thematik, gepaart mit neuen methodischen Ansätzen, belegen. ${ }^{3}$

Die zentrale Anliegen all dieser Arbeiten, ,the banquet as a social institution in its own right in the ancient world" zu betrachten, ${ }^{4}$ stellt auch den Ausgangspunkt für meine Studie dar. Im Folgenden werde ich auf die Bewirtungen als rituelle öffentliche Handlungen fokussieren, deren Mittelpunkt das gemeinsame Konsumieren von

${ }^{1}$ C. Lévi-Strauss, Totemism (Boston 1963), 89.

2 P. Schmitt Pantel, La cité au banquet. Histoire des repas publics dans les cités grecques (Rom 1992), bes. 359-420; s. neuerdings auch P. Schmitt Pantel - F. Lissarague, 'Banquet des cités', in: Thesaurus Cultus et Rituum Antiquorum II (Los Angeles 2004), 239-242.

${ }^{3}$ Vgl. die Arbeiten von D.E. Smith, From Symposium to Eucharist: the Banquet in the Early Christian World (Minneapolis 2003); K.M.D. Dunbabin, The Roman Banquet. Images of Conviviality (Cambridge 2003); K. Vössing, Mensa Regia. Das Bankett beim hellenistischen König und beim römischen Kaiser (München - Leipzig 2004); J.F. Donahue, The Roman Community at Table during the Principate (Ann Arbor 2004); B.K. Gold - J.F. Donahue (Hrsg.), Roman Dining (Baltimore - London 2005); E. Stein-Hölkeskamp, Das römische Gastmahl. Eine Kulturgeschichte (München 2005); K. Vössing (Hrsg.), Das römische Bankett im Spiegel der Altertumswissenschaften (Stuttgart 2008).

${ }^{4}$ Smith 2003, a.a.O. (Anm. 3), 2. 
Essen und Trinken bildete. ${ }^{5}$ Damit sind nicht nur jene öffentlichen Bewirtungsrituale gemeint, die mit Prozessionen, aufwändigen Opfern und Ähnlichem einhergingen, sondern auch solche, die aus einem nicht-kultischen Anlass veranstaltet wurden. Mit dem Begriff ,Handlungen' möchte ich andererseits die Bewirtungen als Teil der politischen und sozialen Praxis in den Poleis interpretieren. Das gemeinsame Mahl war an sich ,politisch', denn war zugleich politisches Instrument und Spiegel der politischen Verhältnisse. ${ }^{6}$ Damit meine ich allerdings nicht, dass es bei den öffentlichen Bewirtungen nur um Macht oder um Machtverhältnisse ging. Ich betrachte sie eher als ein wichtiges - aber keineswegs alleiniges - Feld, auf dem politische Relationen, Konstellationen und Transformationen repräsentiert und manipuliert werden konnten. ${ }^{7}$ Allerdings muss solchen Feststellungen eine genaue Betrachtung und Kategorisierung derjenigen Merkmale vorausgehen, durch die das Phänomen, öffentliche Bewirtung' gekennzeichnet war. ${ }^{8}$

Die öffentlichen Bewirtungen im östlichen Teil des Imperium Romanum (Griechenland und Kleinasien) werden in der Literatur entweder als ein Kontinuum aus der hellenistischen Zeit angesehen und demzufolge undifferenziert kommentiert; oder aber als „GrecoRoman banquets" bezeichnet, ${ }^{9}$ was meines Erachtens eher ein Verlegenheitsterminus ist. Vordergründig betrachtet spricht zwar einiges für eine kontinuierlich ausgeübte Praxis - man bedenke nur die Rolle der Euergeten bei der Finanzierung solcher gemeinsamen Mahlzeiten vor dem Hintergrund der ökonomisch geschwächten Poleis. ${ }^{10}$ Den

5 Vgl. M. Dietler, 'Theorizing the feast: rituals of consumption, commensal politics and power in African contexts', in: M. Dietler - B. Hayden (Hrsg.), Feasts. Archaeological and Ethnographic Perspectives on Food, Politics, and Power (Washington - London 2001), 65-67.

6 Vgl. O.N. van Nijf, 'Review of: P. Schmitt Pantel, La cité au banquet. Histoire des repas publics dans les cités grecques', Journal of Hellenic Studies 114 (1994), 209.

7 Vgl. Dietler 2001, a.a.O. (Anm. 5), 68-71, 75-77.

8 Vgl. Dietler 2001, a.a.O. (Anm. 5), 66 f.

${ }^{9} \mathrm{P}$. Schmitt Pantel, 'Le festin dans la fête de la cité grecque hellénistique', in: F. Dunand (Hrsg.), La fête. Pratique et discours: d'Alexandrie hellénistique à la mission de Besançon (Paris 1981), 85-100; ead. 1992, a.a.O. (Anm. 2); Smith 2003, a.a.O. (Anm. 3); Donahue 2004, a.a.O. (Anm. 3), 48-52. Vgl. van Nijf 1994, a.a.O. (Anm. 6), 209; Vössing 2004, a.a.O. (Anm. 3) 252-264.

${ }^{10}$ Vgl. hierzu die grundlegende Arbeit von F. Quaß, Die Honoratiorenschicht in den Städten des griechischen Ostens. Untersuchungen zur politischen und sozialen Entwicklung in hellenistischer und römischer Zeit (Stuttgart 1993). 
Einfluss Roms macht man hingegen vorwiegend am Kaiserkult fest. ${ }^{11}$ Folglich werden in der Forschung Feste und öffentliche Bewirtungen in den griechischen Poleis weitgehend abgekoppelt von den politischen und sozialen Veränderungen der römischen Epoche betrachtet. Gerade an diesem Punkt möchte ich ansetzen und versuchen aufzuzeigen, dass die römische Herrschaft über den griechischen Osten sehr wohl eine Veränderung der Bewirtungsmodi nach sich gezogen hat. Epigraphisch belegt ist dieser Prozess ab der späthellenistischen Zeit, also etwa ab dem 2. Jh. v. Chr., und er weist eine Reihe von lokalen sowie zeitspezifischen Elementen auf. Einer dieser Belege, der mir als Fallbeispiel für meine Überlegungen dienen soll, ist das bekannte Ehrendekret für Epameinondas aus Akraiphia (IG VII 2712).

\section{Ein Beispiel: Epameinondas aus Akraiphia}

Um 37 n. Chr. führte Epameinondas in der böotischen Stadt Akraiphia eine Reihe von außerordentlichen, ja innovativen Handlungen durch: ${ }^{12}$ Er stiftete ein Fest mit Stieropfern und einem gymnischen Agon zu Ehren von Hermes, Herakles und der Sebastoi (IG VII 2712, Z. 22-25; nach der Berichtigung von J.H. OLIVER); er bewirte-

${ }^{11}$ Vgl. hierzu neuerdings M. Kantiréa, Les dieux et les dieux augustes: le culte impérial en Grèce sous les Julio-Claudiens et les Flaviens. Etudes épigraphiques et archéologiques (Athen - Paris 2007).

${ }^{12} \mathrm{Zu}$ den Ehrendekreten für Epameinondas ( $I G$ VII 2711 und 2712) s. die Berichtigungen und Ergänzungen von J.H. Oliver, 'Epaminondas of Acraephia', Greek, Roman and Byzantine Studies 12 (1971), 221-237 (mit Übersetzung). Vgl. auch L. Robert, 'Études sur les inscriptions et la topographie de la Grèce Centrale. VI. - Décrets d'Akraiphia', Bulletin de Correspondance Hellénique 59 (1935), 438-452 (= id., Opera Minora Selecta I [Amsterdam 1969], 279-293 = SEG 15, 330); M.-H. Quet, 'Remarques sur la place de la fête dans le discours de moralistes grecs et dans l'éloge des cités et des évergètes aux premiers siècles de l'Empire', in: Dunand 1981, a.a.O. (Anm. 9), 52-56; Quaß 1993, a.a.O. (Anm. 10), 312; C. Müller, 'Epaminondas et les euergètes de la cité d'Akraiphia au $1^{\mathrm{er}} \mathrm{s}$. de n. ère', in: A.C. Christopoulou (Hrsg.), 2. Diethnes Synedrio Boiotikon Meleton 1992 II (Athen 1995), 455467; Donahue 2004, a.a.O. (Anm. 3), 50 f. Zu Akraiphia in der späthellenistischen Zeit vgl. J. Ma, 'The many lives of Eugnotos of Akraiphia', in: B. Virgilio (Hrsg.), Studi ellenistici XVI (Pisa 2005), 141-191 (mit der älteren Literatur). S. auch u. Tabelle 1 mit der Auflistung der Anlässe für die Handlungen des Epameinondas und der daran anknüpfenden Bewirtungen. 
te die Bürger von Akraiphia im Gymnasium ${ }^{13}$ und auch die anwesenden Fremden sowie die Kinder von freiem rechtlichen Status und sogar die Sklaven (Z. 25-30); ${ }^{14}$ er veranstaltete ein weiteres Stieropfer zu Ehren der Sebastoi mit anschließender Bewirtung $(\hat{\varepsilon}[\pi \varepsilon] \theta$ oív $\eta \sigma[\varepsilon v])$ der Bürger im Gymnasium (Z. 30-32); er bezahlte die Reparatur eines städtischen Gebäudes aus eigenen Mitteln (Z. 33$37)$; er übernahm freiwillig und aus eigenen Kosten eine Gesandtschaft zu dem neuen Kaiser Caligula in Vertretung des koinon der Böoter (Z. 37-53); und schließlich veranlasste er in seiner Funktion als Agonothet die Wiederaufnahme der pentaeterischen Agone der Megala Ptoia, die seit 30 Jahren nicht mehr durchgeführt worden waren, und stiftete zugleich ein neues Fest, nämlich das der

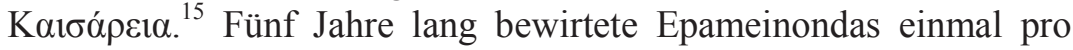
Jahr die Stadt sowie fünfmal im Jahr die städtischen und die BundesMagistrate (Z. 59-63). In dem Jahr, in dem die Agone stattfanden, entfaltete Epameinondas all seine Großzügigkeit, seine Pietät und

${ }^{13} \mathrm{Zu}$ den archäologischen und epigraphischen Belegen für Festmähler in Gymnasia s. E. Mango, 'Bankette im hellenistischen Gymnasion', in: D. Kah - P. Scholz (Hrsg.), Das hellenistische Gymnasion (Berlin 2004), 273-311.

${ }^{14}$ Zur Teilnahme der Sklaven an den öffentlichen Bewirtungen s. Schmitt Pantel 1981, a.a.O (Anm. 9), 92; ead. 1992, a.a.O. (Anm. 2), 399-401 mit weiteren Belegen.

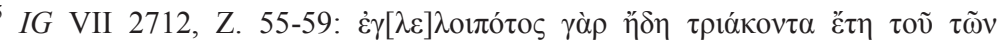

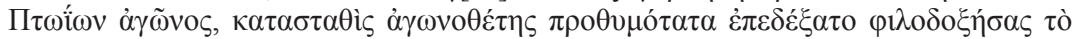

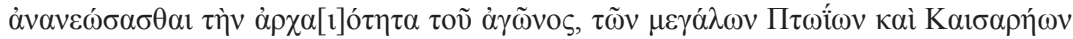

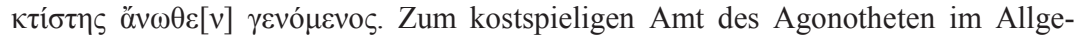
meinen und zu Epameinondas im Besonderen vgl. Quaß 1993, a.a.O. (Anm. 10), 275-285, 305-315. Obwohl die Gründe für den dreißig Jahre lang währenden Ausfall des Festes für Apollon Ptoios in Akraiphia im Inschriftentext nicht ausgeführt werden, ist es doch wahrscheinlich, dass finanzielle Probleme hierbei eine Rolle gespielt haben (dagegen Müller 1995, a.a.O. [Anm. 12], 459). Diese Hypothese lässt sich durch ein weiteres Ehrendekret $(S E G$ 15, 330) der böotischen Stadt aus dem Jahr 42 n. Chr. plausibel machen, in dem berichtet wird, dass es bei der Durchführung der gerade erneuerten Agone für Apollon Ptoios aufgrund von Missernten zu finanziel-

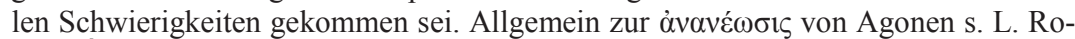
bert, Études anatoliennes (Paris 1937), 426-429; Quaß 1993, a.a.O. (Anm. 10), 284 f. Die Agone der Ptoia sind noch für das 3. Jh. belegt (s. L. Bizard, 'Une inscription du sanctuaire d'Apollon Ptoios trouvée à Larymna', Bulletin de Correspondance Hellénique 27 [1903], 296-299; nach 212 n. Chr.); vgl. auch A. Schachter, Cults of Boiotia (London 1981), 209. Zur Verbindung der althergebrachten Festspiele der Ptoia mit den Kaiserspielen (Kaisareia) vgl. L. Robert, 'Inscriptions d'Athènes et de la Grèce centrale', Archaiologike Ephemeris (1969), 55 (= id., Opera Minora Selecta VII [Amsterdam 1990], 761); allgemein hierzu Quaß 1993, a.a.O. (Anm. 10), 309 f. 
seine Liebe zu seinem Vaterland (Z. 63-66): Seine übliche jährliche Bewirtung der Stadtgemeinde ( sich nun sowohl auf die Bürger als auch auf die Umwohner (paroikoi) und die Gruppe der ektèmenoi. Jeder von ihnen bekam ein Körbchen Weizen und Wein. Er sorgte für eine den Göttern und dem feierlichen Anlass würdige Prozession sowie für die Aufführung des traditionellen Syrtos-Tanzes (Z. 66-67). ${ }^{17}$ Er führte für die Götter und die Sebastoi ein Stieropfer durch und verteilte das Opferfleisch

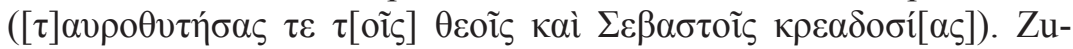
gleich lud er die Söhne aus den besten Familien sowie die erwachsenen Sklaven gruppenweise zu arista (Verabreichungen eines Frühstücks), glykismoi (Bewirtungen mit süßem Wein) und deipna (öffentliche Mahlzeiten) ein (Z. 68-70) ${ }^{18}$ Parallel dazu übernahm seine Frau die Bewirtung der unverheirateten Mädchen und der erwachsenen Sklavinnen (Z. 70-71). Damit aber nicht genug (Z. 71-74): Epameinondas verköstigte auch privat die in Akraiphia zeltenden Besucher und die Schauspieler nach schriftlicher Ankündigung ( $\dot{\alpha} \pi^{\prime}$

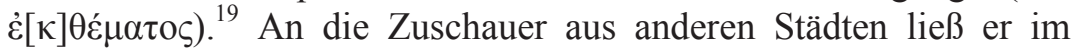
Theater große und mit feinen Zutaten hergestellte Süßigkeiten verteilen. ${ }^{20}$ Nach Beendigung der Agone und der Bewirtung des gesamten

${ }^{16}$ Zum Terminus $\delta$ ió $\delta$ o $\mu \alpha$ s. L. Robert, 'Inscriptions de Didymes et de Milet', in: id., Hellenica 11/12 (1960), 470-474.

${ }^{17}$ Vgl. das Dekret für Soteles aus Pagai (IG VII $190=$ A. Wilhelm, 'Inschrift aus Pagai', Jahreshefte des Österreichischen Archäologischen Instituts 10 [1907], 17-32); Zur Verbindung von Lokalpatriotismus und Euergetismus vgl. Quet 1981, a.a.O. (Anm. 12), 52 f.; A. Chaniotis, 'Sich selbst feiern? Städtische Feste des Hellenismus im Spannungsfeld von Religion und Politik', in: M. Wörrle - P. Zanker (Hrsg.), Stadtbild und Bürgerbild im Hellenismus (München 1995), 161; id., 'Das Bankett des Damas und die Hymnen des Sosandros. Öffentlicher Diskurs über Rituale in den griechischen Städten der Kaiserzeit', in: D. Harth - G. Schenk, (Hrsg.), Ritualdynamik. Kulturübergreifende Studien zur Theorie und Geschichte rituellen Handelns (Heidelberg 2004), 291-304; F. Gascó, 'Evergetismo y conciencia cívica en la parte oriental del Imperio', Habis 26 (1995), 177-186; J.E. Lendon, Empire of Honour. The Art of Government in the Roman World (Oxford 1997), 84-89.

${ }^{18}$ Vgl. u. Anm. 23.

${ }^{19}$ Vgl. I.Priene 111, Z. 175 f.; ibid. 118, Z. 11-15; I.Stratonikeia II 1 (IK 22, 1) 1025, Z. 15-17; SEG 32, 1243 mit dem Kommentar von R. Merkelbach, 'Ehrenbeschluß der Kymäer für den Prytanis Kleanax', Epigraphica Anatolica 1 (1983), 33-38; Vgl. auch Schmitt Pantel 1992, a.a.O. (Anm. 2), 381 f., 402 f.

${ }^{20} I G$ VII 2712, Z. 74-77 mit der Berichtigung von Oliver 1971, a.a.O. (Anm.

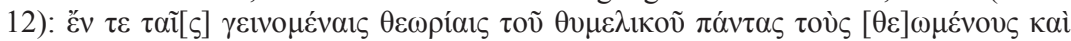

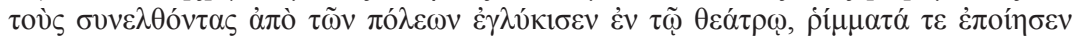

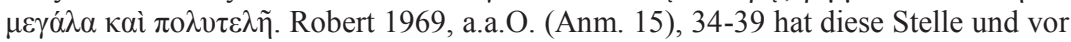


Volkes lud er „oben“, d.h. im Heiligtum des Apollon Ptoios, die Magistrate zu einem kostspieligen Essen (mit altem Wein kata trikleinon $^{21}$ ein. Als Epameinondas anschließend vom Heiligtum in die Stadt zurückkam, wurde er vom Volk erwartet und gefeiert (Z. 8286). Spontan entschied er sich, diese Bekundungen zu erwidern, indem er einen weiteren Stier im Namen der Stadt für Zeus Megistos opferte und anschließend die aus diesem Anlass Versammelten bewirtete.

Faim, piété, goût de l'apparat et de la solennité, plaisir d'être ensemble sous un prétexte, concentration sur une brève période du peu de superflu dont on dispose afin d'en tirer un plaisir maximum en le volatilisant d'un coup: tout cela explique le rythme explosif de la vie collective dans les sociétés pauvres et la place considérable qu'y occupent les banquets.

Mit diesen Worten kommentierte Paul VEYNE das beeindruckende Dekret von Akraiphia. ${ }^{22}$ In der Tat ist in dem Text ein spannend aufgebauter Erzählungsbogen zu erkennen, der in der Schilderung der neu eingeführten Festspiele der Ptoia und Kaisareia seinen Höhepunkt erreicht. Insgesamt sind in dem erhaltenen Teil des Ehrendekrets zwölf öffentliche Bewirtungen beschrieben (sieben davon anlässlich der Ptoia). Fünf unterschiedliche Orte für die Bewirtungen und eine Reihe von Personengruppen als Teilnehmer an diesen Mählern werden aufgezählt. Die gesamte Terminologie des Bankettwesens wird aufgelistet: demothoinia, ariston, deipnon, glykismos, kreadosia. ${ }^{23}$ Im Mittelpunkt steht der Euerget, also Epameinondas selbst, der sich um die Polis verdient gemacht hat. Er hat gestiftet,

allem den Begriff ṕ́ $\mu \mu \alpha \tau \alpha$ untersucht und mit den lateinischen missilia in Verbindung gebracht. Vgl. auch C.P. Jones, 'Dinner Theater', in: W.J. Slater (Hrsg.), Dining in a Classical Context: Contrasts and Parallels (Michigan 1992), $196 \mathrm{f}$.

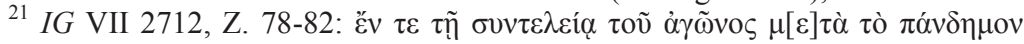

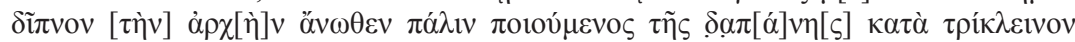

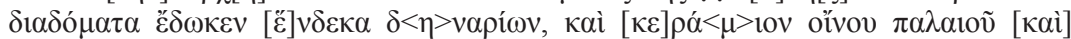

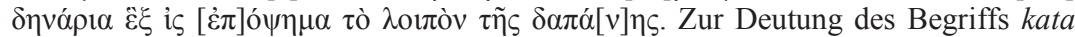
trikleinon als Bezeichnung eines Speisesaales und nicht als Beschreibung des Speisebettes mit drei Plätzen vgl. P. Gauthier, 'Études sur des inscriptions d'Amorgos', Bulletin de Correspondance Hellénique 104 (1980), 215 mit Anm. 55; vgl. auch IG XII 7, 515, Z. 54 f. (Amorgos; Ende 2. Jh. v. Chr.); SEG 35, 744, Z. 20 (Makedonien, Kalindoia; 1. Jh. n. Chr.).

${ }_{22} \mathrm{P}$. Veyne, Le pain et le cirque. Sociologie historique d'un pluralisme politique (Paris 1976), $296 \mathrm{f}$.

${ }^{23}$ Zur Terminologie s. Schmitt Pantel a.a.O. (Anm. 2), 261-289. 
Feste initiiert, mehrfach Opfer durchgeführt, Menschen bewirtet, sei-

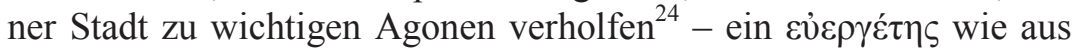
dem Bilderbuch. Aber aus welchem? Aus einem hellenistischen Bilderbuch ist Epameinondas jedenfalls nicht entsprungen. Vielmehr ordnet er sich ein in eine Reihe von Euergeten, die unter neuen Rahmenbedingungen und durch neuartige Anforderungen geformt wurden.

\section{Zur Typologie der öffentlichen Bewirtungen in der Kaiserzeit}

Möchte man die öffentlichen Bewirtungen der klassischen und hellenistischen Zeit mit einigen Schlagworten kennzeichnen, so würde man wohl am ehesten auf Formulierungen zurückgreifen wie „gleiche Portionen für alle Bürger"; oder aber auf den Ausdruck ouk apophora, dem ein ,Zwang' zum gemeinsamen Essen und Trinken an einem bestimmten Ort zu Grunde lag. ${ }^{25}$ Bei dieser Art von Bewirtungen waren die Rollen des Gastgebers und der Gäste sowie die Form der Reziprozität von vornherein festgelegt. Als Gastgeber trat die Stadt auf, und der Kreis der Gäste bestand aus der exklusiven Gruppe der männlichen Polis-Bürger. Die Polis verteilte das Opferfleisch, während sich die Bürger durch die Ausübung ihrer Bürgerpflichten dazu verpflichteten, dieses, Geschenk ${ }^{6}$ zu erwidern. Die Gemeinschaft sah sich durch einen solchen Vorgang bestätigt und reproduzierte sich zugleich auf eine symbolische Art und Weise. ${ }^{26}$

${ }^{24}$ Wie Quet 1981, a.a.O. (Anm. 12), 54, bezüglich der durchgehenden Verwendung der dritten Person im Singular richtig bemerkt: "l'évergète agonothète apparaît comme le seul dispensateur de la fête $[\ldots]$. Aucune commission désignée par la cité n'est mentionnée dans le texte".

${ }^{25}$ Vgl. N. Loraux, 'La cité comme cuisine et comme partage', Annales. Economies, Sociétés, Civilisations 36 (1981), 614-622; P. Schmitt Pantel, 'Banquet et cité grecque', Mélanges de l'École Française de Rome - Antiquité 97 (1985), 154-157; ead. 1992, a.a.O. (Anm. 2), 247-252; P. Garnsey, Food and Society in Classical Antiquity (Cambridge 2002), 131-134; Schmitt Pantel - Lissarague 2004, a.a.O. (Anm. 2), $241 \mathrm{f}$. Zur Bedeutung des vor allem in den Opferkalendern belegten Ausdrucks ouk (apo)phora, der wohl zur Verlängerung, Verstärkung und Hervorhebung des kultischen Aspekts des Tieropfers und der sich daran anschließenden gemeinsamen Mahlzeit diente, vgl. die Diskussion bei G. Ekroth, The Sacrificial Rituals of Greek Hero-Cults (Liège 2002), 321-325.

${ }^{26}$ Zur integrierenden und stabilisierenden sozialen Funktion von Festen vgl. F. Dunant, 'Sens et fonction de la fête dans la Grèce hellénistique. Les cérémonies en l'honneur d'Artémis Leucophryene', Dialogues d'Histoire Ancienne 4 (1978), 203229; C. Calame, 'Morfologia e funzione della festa nell'antichità', Annali dell'Istitu- 
Die Vielzahl von Bewirtungen und die Vielfalt an Personengruppen, die im Dekret für Epameinondas Erwähnung finden, führen uns eine Reihe von fragmentarischen Bildern vor Augen, die man zusammensetzen muss, um zu einem kollektiven Bild der Polis zu gelangen. Darüber hinaus erscheinen unter den genannten Personengruppen auch solche, die man nicht der Bürgerschaft zugerechnet haben dürfte, wie etwa Fremde, Sklaven, Kinder oder sogar zugereiste Händler. ${ }^{27}$ Dabei ist eine ständige Inklusion und Exklusion zu beobachten, was dem gemeinsamen Feiern jedoch keinen Abbruch tat. Die Stellung des Epameinondas als Gastgeber im eigenen Interesse oder als Gastgeber im Namen der Polis scheint das verbindende Element bei all diesen öffentlichen Bewirtungen im kleinen oder größeren Kreis gewesen zu sein. Was sich allerdings hinter dem integrativen Element der generösen Gastfreundschaft, die der Person des Epameinondas zugeschrieben wird, verbirgt, sind die relativen und asymmetrischen Verhältnisse zwischen den einzelnen Gruppierungen. Denn die öffentlichen Bewirtungen wurden genauso wie andere Feiern zwar von den Teilnehmern als harmonische Zelebrierung der gemeinsamen Identität und der Eintracht empfunden, waren zugleich aber auch Schauplätze, in denen um soziales Prestige, um politischen Einfluss, um informelle Macht, also kurz gesagt um symbolisches Kapital gerungen wurde. ${ }^{28}$ Solidarität und Wettbewerb schlossen sich somit keineswegs aus, sondern sie kennzeichneten geradezu die den Bewirtungen inhärente Polysemie. ${ }^{29}$ Sie trennten und vereinigten die Bürgerschaft gleichermaßen, genauso wie sie sowohl Identitätsbildungen als auch Grenzziehungen bewirkten. Die politi-

to Universitario Orientale di Napoli - Sezione Filologico-Letteraria 4/5 (1982/83), 3-23; Chaniotis 1995, a.a.O. (Anm. 17), 145-172.

${ }^{27}$ Unterschiedliche Teilnehmergruppen, die entweder am gleichen Tag oder auf verschiedene Tage verteilt bewirtet wurden, sind im epigraphischen Material bereits seit dem 2. Jh. v. Chr. zu fassen: $I G$ XII 7, 515 (Aegiale, Amorgos); I.Sestos (IK 19) 1 (= OGIS 339; ca. 125 v. Chr.); IG XII 9, 234 (Eretria; ca. 100 v. Chr.); Athenische Mitteilungen 35 (1910), 409f. Nr. 3; IGR IV 294 (Pergamon; 1. Hälfte 1. Jh. v.Chr.); I.Priene 113 (1. Jh. v.Chr.); I.Stratonikeia I (IK 21) 170 (Panamara, claudisch); I.Stratonikeia II 1 (IK 22, 1) 1025 (Karien, antoninisch); vgl. hierzu P. Gauthier, 'Notes sur le rôle du gymnase dans les cités hellénistiques', in: Wörrle - Zanker 1995, a.a.O. (Anm. 17), 9. Zur Differenzierung bzw. Hierarchisierung der Teilnehmergruppen s. Schmitt Pantel 1981, a.a.O. (Anm. 9), 91-93; ead. 1992, a.a.O. (Anm. 2), 380-408; sowie weiter u. im Text.

28 Vgl. Dietler 2001, a.a.O. (Anm. 5), 76-78.

29 Vgl. Dietler 2001, a.a.O. (Anm. 5), 77 f. 
sche Arena, in der sich Epameinondas und die vor ihm und nach ihm kommenden griechisch-römischen Euergeten bewegten, war die der öffentlichen Bewirtungen. In dieser Arena wurde das Verhältnis der Elite zum Volk, zur Polis und zu den eigenen Standesgenossen ausgehandelt. ${ }^{30}$ Die Euergeten traten hierbei mit dem Volk in Kontakt, bewahrten dafür aber gebührenden Abstand; sie konkurrierten mit ihren Standesgenossen, indem sie mit ihnen um den Rang des größten und des exklusivsten Stifters wetteiferten; sie übernahmen die Rolle des Patrons ihrer Stadt, indem sie ihr dazu verhalfen, als die attraktivste aller Poleis zu gelten. ${ }^{31}$

Die zwölf Bewirtungen, die Epameinondas veranlasst hat, lassen exemplarisch diese verschiedenen Ebenen erkennen. Ich möchte sie in Anlehnung an die analytischen Konzepte von Michael DIETLER zu Festen und Bewirtungen in drei Typen unterteilen: die ,empowering'-Feste, die ,patron-role'-Feste und zuletzt die ,diacritical'Feste. ${ }^{32}$ Bei den ,empowering 6 -Festen geht es um den Erwerb und

${ }^{30}$ Quet 1981, a.a.O. (Anm. 12), 55 verweist darauf, dass die im Dekret auftauchenden Termini polis und dèmos ,ne renvoient pas toujours à une communauté politique, mais à une collectivité plus large qui regroupe les sexes, les classes d'âge, et les catégories juridiques: la foule même que Dion de Pruse et Plutarque désignent par les termes de $\tau$ ò $\pi \lambda \tilde{\eta} \theta 0 \varsigma$, oi $\pi \mathrm{o} \lambda \lambda \mathrm{oí}^{\prime \prime}$.

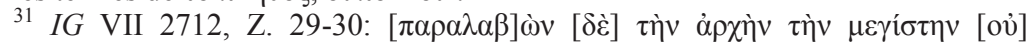

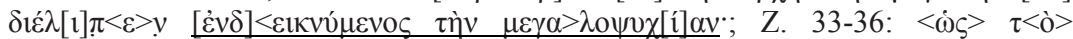

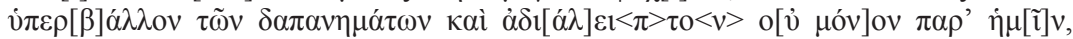

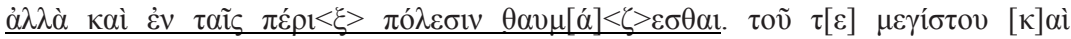

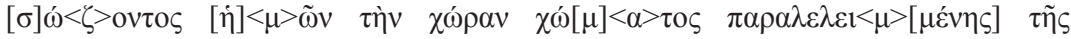

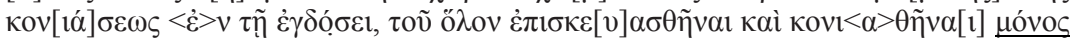

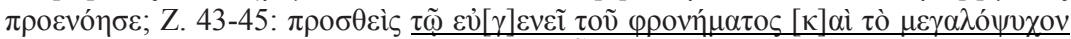

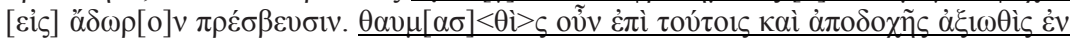

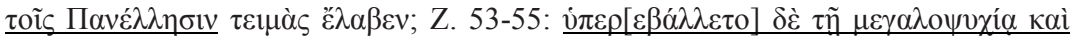

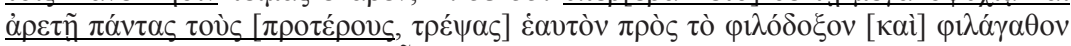

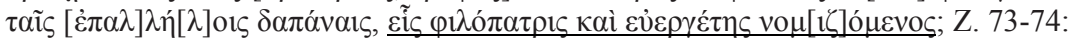

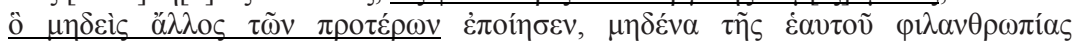

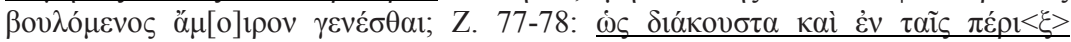

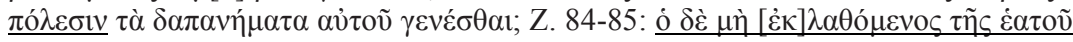

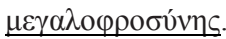

32 Dietler 2001, a.a.O. (Anm. 5), 65-114, bes. 75-88. J. Donahue hat mit Hilfe moderner soziologischer Kategorien nach Claude Grignon (,,institutional, domestic, exceptional, segregative, transgressive") eine Typologie der öffentlichen römischen Bankette vorgeschlagen und auf drei Charakteristika aufmerksam gemacht: Die außergewöhnliche Kommensalität (,exceptional commensality“) in Verbindung mit kultischen oder säkularen Festen im Jahreszyklus; die abgrenzende Kommensalität (,segregative commensality“), wie sie in den Mahlzeiten der collegia zu beobachten ist; und schließlich die grenzüberschreitende Kommensalität (,transgressive com- 
die Bewahrung von symbolischem und manchmal sogar von ökonomischem Kapital durch Einflussnahme auf die Bewirtungen. Beim zweiten Typus werden Bewirtungen dafür verwendet, um institutionalisierte, sozial asymmetrische Verhältnisse symbolisch zu visualisieren und dadurch zu verfestigen sowie zu perpetuieren. Der dritte Typus der, diacritical'-Feste beinhaltet den Einsatz von besonderen, exklusiven Gütern oder eines besonderen Konsumstils, um dadurch Rangunterschiede innerhalb bestimmter sozialer Gruppen symbolisch zum Ausdruck zu bringen. Im Unterschied zum zweiten Typus, der ebenfalls auf die Betonung des asymmetrischen Verhältnisses zwischen Gastgeber und Gast abzielt, liegt hier der Akzent nicht auf der Quantität der Bewirtung, sondern auf Fragen des Geschmacks und des Stils.

\section{Monos kai prōtos: Öffentliche Bewirtungen und symbolisches Kapital}

Die Stiftung eines agōn gymnikos mit anschließendem, aufwändigem Stieropfer für die Götter Hermes, Herakles sowie für die Kaiser (Sebastoi), das Stieropfer anlässlich der Amtseinführung des Epameinondas, welches ebenfalls den Kaisern gewidmet war, sowie die Wiedereinführung der Agone der Großen Ptoia und die Neueinführung der Kaisareia, die mit einer Reihe von Bewirtungen einhergingen, haben Epameinondas zweifellos ein hohes symbolisches Anse-

mensality") bei einem sozial abgestuften Gästekreis: J.F. Donahue, 'Toward a typology of Roman public feasting', American Journal of Philology 124 (2003), 423-441 (= in: Gold - Donahue 2005, a.a.O. [Anm. 3], 95-113). Das Ziel von C. Grignon, 'Commensality and social morphology: an essay of typology', in: P. Scholliers (Hrsg.), Food, Drink, and Identity: Cooking, Eating, and Drinking in Europe since the Middle Ages (Oxford 2001), 23-33 - und im Anschluss daran der Typologie von Donahue - ist ,to outline a reasoned inventory of commensal types" (S. 25), die wiederum „a result and a manifestation of a pre-existing group“ (S. 24) darstellen. Das Problem dabei ist, dass keine weiteren Determinanten für die Definition von ,Gruppe' geliefert werden - etwa dazu, wie sich eine solche konstituiert oder wie sie sich von anderen Gruppen unterscheidet (vgl. P. Bourdieu, 'What makes a social class? On the theoretical and practical existence of Groups', Berkeley Journal of Sociology 32 [1982], 1-17). Dennoch sind die Typologien von Dietler 2001 und von Grignon 2001 durchaus miteinander kombinierbar; der Unterschied besteht lediglich darin, dass die Typologie von Dietler nicht nur auf die Interpretation der unterschiedlichen Mahlgemeinschaften anwendbar ist, sondern vor allem auf das Verhältnis zwischen Mahlgemeinschaften und (politischer/sozialer) Macht fokussiert. 
hen eingebracht: „Er hat als erster in der gesamten Menschheitsgeschichte die Verleihung von Schilden als Preise bei den Agonen er-

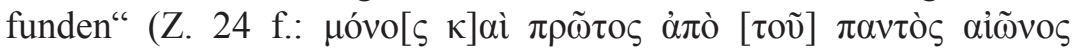

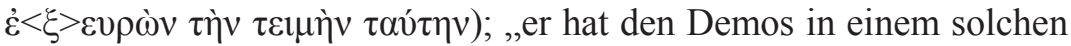
$\mathrm{Ma} ß$ bewirtet, dass die Poleis in der ganzen Region neidisch auf Akraiphia schauten" (Z. 32-34: $<\dot{\omega} \varsigma>\quad \tau<\dot{\mathrm{o}}>\quad \dot{v} \pi \varepsilon \rho[\beta] \dot{\alpha} \lambda \lambda \mathrm{ov} \quad \tau \tilde{\omega} v$

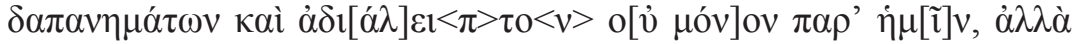

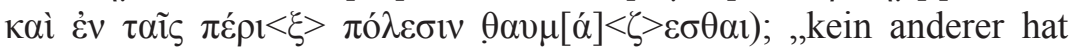
solch große und exquisite Geschenke verteilt, von denen auch in den

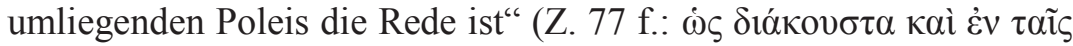

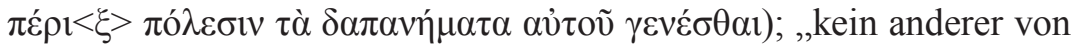
den vorherigen Agonotheten hat so (eine Bewirtung) veranlasst" (Z.

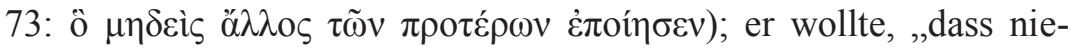
mand an seiner Philanthropie ohne Anteil (amoiros) bliebe“" (Z. 74:

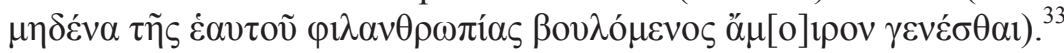

Epameinondas hat also bereits existierende Feste und Bewirtungen mit neuen Elementen versehen, traditionelle, aber vergessene Feste und Gebräuche wiedereingeführt und besonders aufwendige Opfer organisiert. Er hat den Kaiserkult mit dem Kult für Apollo Ptoios assoziiert, was wiederum auf seine persönliche Verbundenheit mit dem römischen Staat hinweist. ${ }^{34}$ Er hat sich als einziger unter den vielen ,vornehmen und erstrangigen“ Männern aus den größeren

${ }^{33}$ Die einzigartigen Verhaltensweisen und Leistungen des Epameinondas werden darüber hinaus durch Termini wie megalopsychia, philopatria, pronoia zum

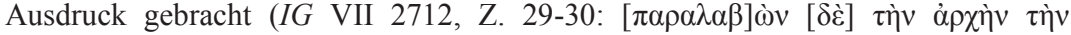

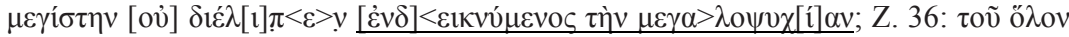

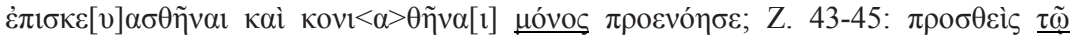

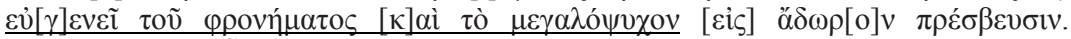

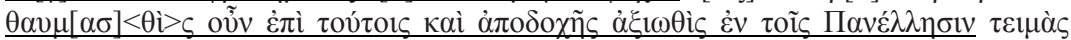

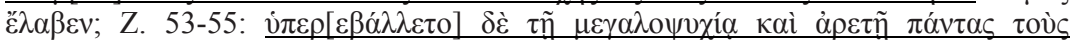

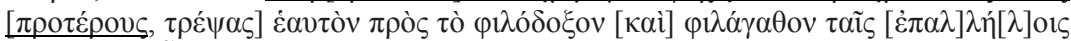

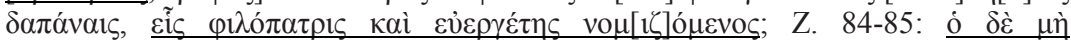

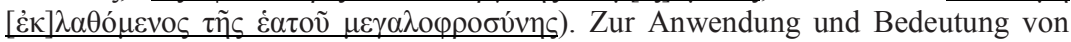

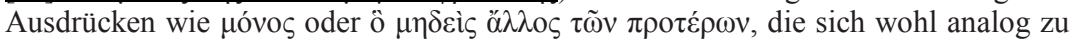
den in den kaiserzeitlichen Inschriften erwähnten Adjektiven singularis und unicus verhalten, vgl. G. Alföldy, Die Rolle des Einzelnen in der Gesellschaft des Römischen Kaiserreiches: Erwartungen und Wertmaßstäbe (Heidelberg 1980), bes. 17 f., 34-38 mit weiteren Beispielen aus dem griechischsprachigen Kulturraum. Die Belege in dem vorliegenden Ehrendekret für Epameinondas sind allerdings - soweit ich sehe - früher als diejenigen aus dem kleinasiatischen Raum. Vgl. auch T.R. Stevenson, 'Social and psychological interpretations of Graeco-Roman religion: some thoughts on the ideal benefactor', Antichthon 30 (1996), 1-18.

${ }^{34}$ S.u. Anm. 35. 
Städten Böotiens bereit erklärt, das koinon der Böoter bei der Gratulationsgesandtschaft der Panhellenen zum Regierungsantritt des Kaisers Caligula zu vertreten. ${ }^{35}$ Die Hervorhebung des Kaiserkultes durch die drei Stieropfer ist ein subtiles Indiz sowohl für die persönliche politische Macht des Epameinondas als auch für den Versuch, Akraiphia als besonders loyale Stadt unter den böotischen Poleis zu etablieren. ${ }^{36}$

Die Wiedereinführung des Festes der Ptoia erforderte eine genaue Planung, aber auch die Investition von Zeit und Arbeit sowie die Bereitstellung von Essen und Getränken seitens des Gastgebers, also seitens der Stadt Akraiphia. Auch in diesem Fall erwies sich Epameinondas als genialer Organisator. ${ }^{37}$ Er mobilisierte nicht nur die Einwohner von Akraiphia, indem er an die Bürger, die paroikoi und die Gruppe der ektêmenoi reichlich Essen verteilte und sie damit zu aktiver Mitarbeit motivierte, sondern auch die lokalen Bäcker, Metzger und Krämer. Die „großen und luxuriösen“ Geschenke, die den Zuschauern und Besuchern aus anderen Städten im Theater übergeben wurden, ließ er aus eigenen Mitteln herstellen. Damit kur-

${ }^{35} I G$ VII 2711; ibid. 2712, Z. 37-55; Vgl. Quaß 1993, a.a.O. (Anm. 10), 173 f;; A. Chaniotis, 'Der Kaiserkult im Osten des Römischen Reiches im Kontext der zeitgenössischen Ritualpraxis', in: H. Cancik - K. Hitzl (Hrsg.), Die Praxis der Herrscherverehrung in Rom und seinen Provinzen (Tübingen 2003), 10 (zu Epameinondas); K. Harter-Uiobopuu, 'Kaiserkult und Herrscherverehrung in den Koina des griechischen Mutterlandes', in: ibid., 216 f. (zu Epameinondas und dem koinon der Böoter), 222 f. (zu Epameinondas und den Panachäern). In seiner späteren Stellung als städtischer Kaiserkultpriester beantragte Epameinondas einen Beschluss zu Ehren des Kaisers Nero anlässlich von dessen Deklaration der Freiheit und Autonomie für die griechischen Städte im Jahre 67; s. IG VII 2713 III, Z. 27-58 (= Syll ${ }^{3} 814$ ); C.P. Jones, 'Nero speaking', Harvard Studies in Classical Philology 100 (2000), 459.

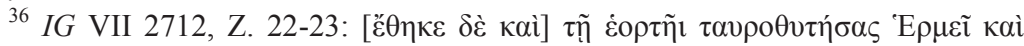

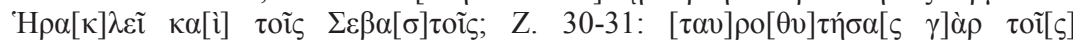

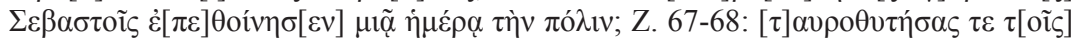

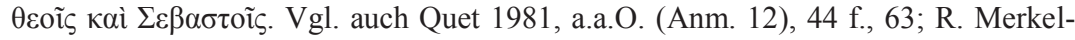
bach, 'Der Rangstreit der Städte Asiens und die Rede des Aelius Aristeides über die Eintracht', Zeitschrift für Papyrologie und Epigraphik 32 (1978), 287-296; I. Maupai, Die Macht der Schönheit: Untersuchungen zu einem Aspekt des Selbstverständnisses und der Selbstdarstellung griechischer Städte in der römischen Kaiserzeit (Bonn 2003).

${ }^{37}$ Vgl. Dietler 2001, a.a.O. (Anm. 5), 79-82, bes. 79: „Commensal hospitality may be manipulated in the empowering feast pattern for economic advantage as well as for political power, especially through the institution of the work feast"; s. auch Donahue 2004, a.a.O. (Anm. 3), 24 f. zur Vorbereitung von römischen Bewirtungen. 
belte er nicht nur die Wirtschaft der Polis an, sondern seine Großzügigkeit bereitete auch den Boden für den ökonomisch gesicherten Weiterbestand des Festes. Eine aus der Zeit des Claudius stammende Inschrift aus Akraiphia bezeugt die enormen finanziellen Schwierigkeiten der Stadt, aus eigenen Mitteln das mit dem Fest der Ptoia verbundene Opfer für Apollon und den Kaiser zu bestreiten. Sie weist nochmals auf die Bedeutung der Organisation eines solchen großen Festes hin: ${ }^{38}$ Der Beitrag der drei geehrten Bürger bestand darin, die Kleinhändler, die Metzger und die Bäcker, „die gewohnt sind, nach

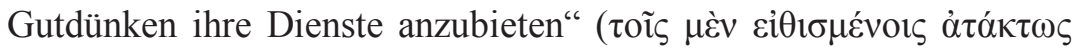

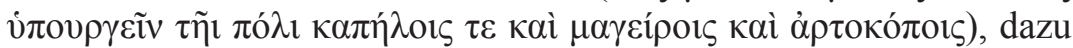
zu bewegen, sich an dem Fest zu beteiligen. Gelungen ist ihnen dies durch die Belieferung der Bäcker mit Weizen bzw. durch die Vergabe von zinslosen Darlehen. ${ }^{39}$

Das große, überregionale Fest mit all seinen Bewirtungen und Opfern war also ein öffentliches rituelles Spiel, eingebettet in kontinuierlich ablaufende politische, ökonomische und soziale Aushandlungsprozesse. Es propagierte die breite und uneingeschränkte Unterstützung aller Teilnehmer für die Realisierung des Festes, die der Gastgeber durch verschiedene Aktionen herbeizuführen vermocht hatte. Somit wurde der Anschein erzeugt, dass die Teilnehmer dem Ganzen nicht passiv beiwohnten. Vor allem aber bot das Fest dem Gastgeber und Euergeten die Möglichkeit, sich und seine ökonomische Überlegenheit in diesem Kontext zur Schau zu stellen, ohne den Neid der Massen zu provozieren. ${ }^{40}$ Zwar werden in der Inschrift die großzügigen Aufwendungen des Epameinondas aufgeführt, jedoch immer in engem Bezug zu seinen sozialen und moralischen Qualitäten, als ob die Prahlerei über die Ausgaben zugleich Zeugnis für seine Person ablegen könne: Attribute wie etwa die „Liebe zur Heimat“

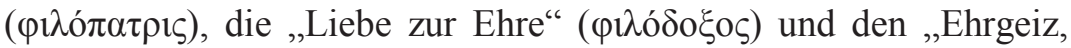

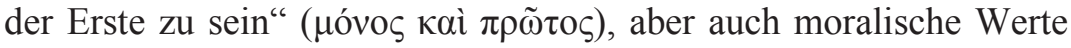

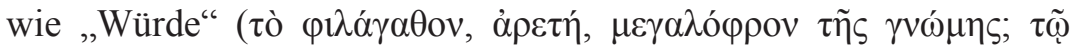

${ }^{38}$ SEG 15, 330 = Robert 1935, a.a.O. (Anm. 12).

${ }^{39}$ SEG 15, 330, Z. 51-61 mit den Bemerkungen von Robert 1935, a.a.O. (Anm. 12), $448 \mathrm{f}$.

${ }^{40}$ Man könnte dies auch als 'Verkennung' ('méconnaissance') nach P. Bourdieu bezeichnen, vgl. id., Sozialer Sinn: Kritik der theoretischen Vernunft (Frankfurt a.M. 1987), 123 f., 193 f. ("der Gabentausch [...] funktioniert, wenn die Wahrheit des objektiven 'Tauschmechanismus' individuell und kollektiv verkannt wird"). S. auch Dietler 2001, a.a.O. (Anm. 5), 76. 


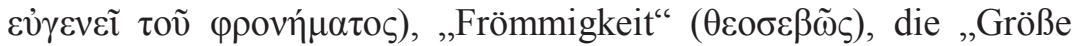
der Seele“ ( $\mu \varepsilon \gamma \alpha \lambda$ ó $\psi v \chi 0 v)$ oder „Großzügigkeit" ( werden nebeneinander aufgeführt. Aufgrund all dieser Tugenden wurden seine Aufwendungen von seinen Mitbürgern nicht als beleidigend empfunden, ${ }^{41}$ sondern sie verpflichteten ihn im Gegenteil sogar, diese Position innerhalb der Gemeinde einzunehmen und infolgedessen das Fest nach eigenem Ermessen zu gestalten.

\section{Mēdena paralipōn: Öffentliche Bewirtungen und die Konstruktion von Autorität}

Bewirtungen sind oft durch Reziprozität gekennzeichnet, aber in unserem Fallbeispiel treffen wir auf solche, bei denen eine Gegenleistung gar nicht zu erwarten war. ${ }^{42}$ Auf der einen Seite standen dabei diejenigen Personengruppen, die ihre immerwährende Rolle als Gäste und somit ihren untergeordneten Status dem Gastgeber gegenüber symbolisch zu akzeptieren hatten. Auf der anderen Seite wurde die Rolle des permanent großzügigen Gastgebers als eine Pflicht betrachtet, die eben aus seiner gehobenen sozialen Stellung beziehungsweise aus seiner politischen Position erwuchs. Eine solche Kommensalität unterstrich die asymmetrische Beziehung zwischen ungleichen Partnern, die eher als ein Verhältnis zwischen Patron und Klient zu definieren ist. Was aus der Institutionalisierung eines so gearteten Bewirtungsmodus folgte, war die Institutionalisierung der Autorität und des Patrons (oder des ,Überpolites' nach Michael WÖRRLE). ${ }^{43}$

Die Schlüsselszene im Dekret für Epameinondas ist bezeichnend hierfür: Nach Beendigung der Festspiele der Ptoia und Kaisareia und im Anschluss an die Bewirtung der Magistrate „oben“ (im Heiligtum des Apollon), kommt Epameinondas nun nach „unten“ in die Stadt und wird von dem gesamten Demos jubelnd empfangen. ${ }^{44}$ Die-

${ }^{41}$ Plutarch, Moralia 813B; 823B; mit dem Kommentar von Quet 1981, a.a.O. (Anm. 12), 48.

${ }^{42}$ Vgl. Dietler 2001, a.a.O. (Anm. 5), 83.

${ }^{43}$ M. Wörrle, 'Vom tugendsamen Jüngling zum "gestressten” Euergeten. Überlegungen zum Bürgerbild hellenistischer Ehrendekrete', in: Wörrle - Zanker 1995, a.a.O. (Anm. 17), 244.

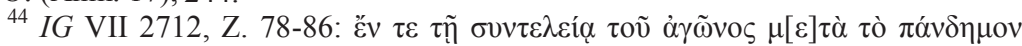

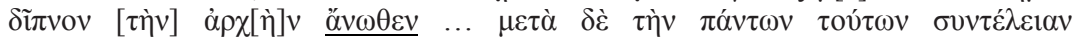

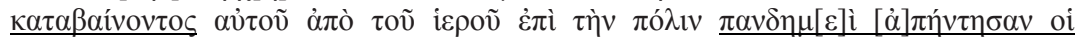


se Dankesbezeugung ,zwingt“ ihn zu einem letzten, spontanen Stieropfer an Zeus Megistos und zu einer nochmaligen Bewirtung diesmal des ganzen Volkes. Das Verhältnis zwischen Epameinondas und dem Volk hätte man nicht plastischer schildern können: „Oben“ im Heiligtum speiste Epameinondas zusammen mit den anderen Magistrate, während ,unten“ in der Polis die Volksmenge auf ihn wartete, um ihm zuzujubeln. Der Dialog zwischen Epameinondas und seinen Anhängern endete also mit einer erneuten Bestätigung des sozialen Status beider Seiten - hier des Patron, dort der Klienten - durch ein Bewirtungsritual. ${ }^{45}$ Innerhalb dieses asymmetrischen Verhältnisses war die Gemeinde der inaktive Partner. Ihre Rolle wird auch nicht weiter präzisiert: Die Festteilnehmer sind im Theater versammelt und nehmen in verschiedenen Gruppierungen an den Bewirtungen teil. Aktiv werden sie nur, wenn sie sich beim Gastgeber bedanken, wie die gerade angesprochene Szene zeigt. Lediglich dieser Part ist für sie bestimmt - die Rolle des dankbaren und zufriedenen Gastes.

Auch das Zelebrieren der communitas wurde immer durch die Handlungen des Epameinondas veranlasst. Die Festgemeinde feierte sich zwar nicht als eine politische Gemeinschaft, wie die verschiedenen sich außerhalb der Bürgerschaft befindlichen Teilnehmergruppen demonstrieren, doch war das Gefühl einer Zusammengehörigkeit dadurch nicht weniger ausgeprägt, ,puisque toute la population communie par l'oreille, les yeux, la bouche, dans le partage de nourriture, de spectacles, de sons, dans les lieux privilégiés et en un temps consacré"“. ${ }^{46}$ Keiner sollte von dieser neu geschaffenen Gemeinschaft ausgeschlossen sein. Nicht nur die Magistrate, die Bürger, die ansässigen Fremden, die paroikoi sowie die Sklaven der Bürger, sondern auch diejenigen, die während der Agone ihre Zelte in Akraiphia auf-

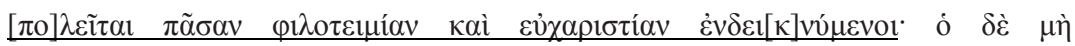

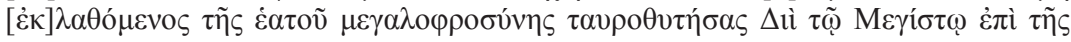

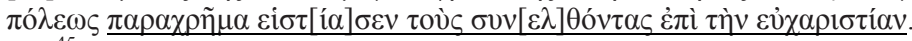

${ }^{45}$ Vgl. die Bemerkungen von Grignon 2001, a.a.O. (Anm. 32), 31 f. zu dieser Art von grenzüberschreitender Kommensalität, deren Hauptcharakteristikum die Ambivalenz ist: „It is because it recognises these borders that it can temporarily and symbolically transgress them, providing an opportunity for establishing, in the neutralised and ritual parenthesis of a meal, a relation of exchange. And it is by transgressing them that it contributes to recognising and maintaining them". Vgl. hierzu auch Schmitt Pantel 1981, a.a.O. (Anm. 9), 92.

${ }^{46}$ Quet 1981, a.a.O. (Anm. 12), 61. 
schlugen, hat Epameinondas bei sich zu Hause bewirtet, ${ }^{47}$,denn er wollte, dass alle an seiner philanthropia teilhaben". ${ }^{48}$ Da die Besucher nicht zu einer der Gruppen gehörten, die zu einer Bewirtung oder Essensverteilung eingeladen wurden, ${ }^{49}$ hat er sich ihrer erbarmt und durch eine private Einladung seine Erhabenheit und Großzügigkeit zur Schau gestellt. Epameinondas erwies sich somit erneut als derjenige, der nicht nur das Fest veranstaltete, sondern auch die Stellung der einzelnen Teilnehmer innerhalb dieses Rahmens bestimmte.

\section{Oinos palaios: Öffentliche Bewirtungen und die Konstruktion der Exklusivität}

Die (politische) Polysemie der öffentlichen Bewirtungen ermöglichte allerdings nicht nur die Konstruktion einer vertikalen Differenzierung mit gleichzeitiger Festlegung der Beziehungen zwischen Gastgeber und Gästen, sondern auch die Positionierung eines Gastgebers innerhalb seiner eigenen gesellschaftlichen Gruppe. ${ }^{50}$ Zwar wurde auch durch diesen Bewirtungsmodus die Vergegenständlichung eines ungleichen sozialen Verhältnisses angestrebt, wie es bereits beim vorigen Modus der Fall war, doch unterscheidet er sich von diesem in einigen wichtigen Aspekten. Zum einen ist eine Verschiebung in der Symbolik der Bewirtungen festzustellen: Im Mittelpunkt standen nun nicht mehr die Quantität beziehungsweise die Großzügigkeit des Gastgebers, sondern der Stil und die Qualität der Bewirtung. Darüber hinaus ist eine weitere Verschiebung von einem sich auf die Kommensalität stützenden hierarchischen Verhältnis zwischen ungleichen Partnern hin zu einer Bildung von exklusiven Bewirtungskreisen zu beobachten, innerhalb derer die Erwiderung der Gastfreundschaft keine Rolle mehr für Status- oder Machtkonstruktionen spielte.

Die Mahlzeiten, die Epameinondas und seine Frau für die Söhne und Mädchen der „Besten der Bürger“ anboten, sowie die Bewirtung

${ }^{47}$ Vgl. I.Priene 109, Z. 177-179, 192-194; s. auch Quet 1981, a.a.O. (Anm. 12), 56, die von einer schleichenden Konfusion zwischen dem öffentlichen (politischen und kultischen) Raum und dem privaten Raum - d.h. dem Haus des Agonothetes bzw. des Euergetes - spricht.

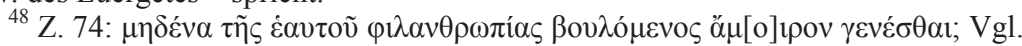

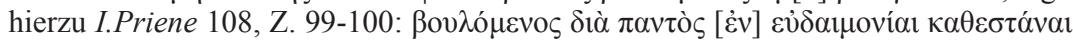

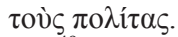

${ }^{49}$ S.o. Anm. 14 und 27.

${ }^{50}$ Vgl. Dietler 2001, a.a.O. (Anm. 5), 85 f. 
für die archē im Heiligtum des Apollon Ptoios sind Beispiele hierfür. ${ }^{51}$ Im ersteren Falle ist interessant, dass die Einladung des Ehepaares allein Bürgern und deren Sprösslingen aus den besten gesell-

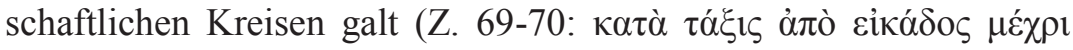

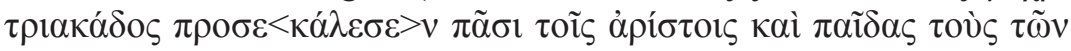
$\pi \circ \lambda \iota \tau \tilde{\omega} v)$. Es wurden also nicht etwa alle Bürger der Stadt zum Essen eingeladen, sondern nur diejenigen, die den gleichen Status wie Epameinondas besaßen. Bei diesem Anlass erscheint zum ersten Mal die Ehefrau von Epameinondas als Gastgeberin, die für die Bewirtung des Nachwuchses des eigenen Geschlechts Verantwortung übernimmt. ${ }^{52}$ Diese Bewirtungen zielten somit auf die Repräsentation des Ehepaares und seine Stilisierung innerhalb der eigenen sozialen Schichten ab und definierten zugleich ihre Zugehörigkeit zur Elite. Dadurch bildete sich ein exklusiver Kreis, der sich eindeutig von den anderen gesellschaftlichen Gruppierungen abgrenzen ließ.

Stil oder Geschmack konnten ebenfalls als Zeichen der Distinktion eingesetzt werden, um eine Personengruppe von den anderen zu

${ }^{51}$ Zur Privilegierung von politischen Gremien oder Gruppierungen, die aus Mitgliedern der städtischen Oberschichten bestanden, s. beispielsweise A. Balland, Fouilles de Xanthos VII. Inscriptions d'époque impériale du Létôon (Paris 1981), 211 f.; G. Woolf, 'Food, poverty and patronage. The significance of the epigraphy of the Roman alimentary schemes in early imperial Italy', Papers of the British School at Rome 58 (1990), 214; Schmitt Pantel 1992, a.a.O. (Anm. 2), 387 f.; P. Herz, 'Fest und Gemeinde: Feiern des Kaiserkultes und die Gemeinschaft der Bürger', Die alte Stadt. Vierteljahreszeitschrift für Stadtgeschichte, Stadtsoziologie und Denkmalpflege 22 (1995), $74 \mathrm{f}$.

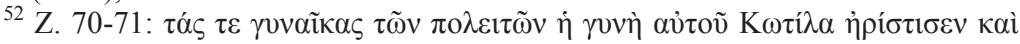

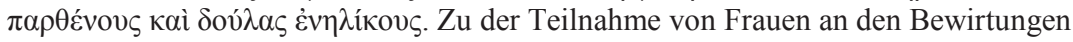
der hellenistischen Epoche und der Kaiserzeit vgl. Schmitt Pantel 1981, a.a.O. (Anm. 9), 92; ead. 1992, a.a.O. (Anm. 2), 379 f., 397-399, die von einem Ausschluss der Frauen spricht; dagegen R. van Bremen, The Limits of Participation: Women and Civic Life in the Greek East in the Hellenistic and Roman Periods (Amsterdam 1996), 150-155; E. Stavrianopoulou, Gruppenbild mit Dame: Untersuchungen zur rechtlichen und sozialen Stellung der Frau auf den Kykladen im Hellenismus und in der römischen Kaiserzeit (Stuttgart 2006), 205-208, 212-219 (mit den Belegen von den Kykladen). Sowohl auf den Kykladen als auch in mehreren Städten des kleinasiatischen Raumes (z.B. in Didyma, Lagina, Panamara) ist bei Bewirtungen eine ähnliche ,Genderverteilung' zwischen Eheleuten oder Vätern und Töchtern, die gemeinsam als Veranstalter auftraten, zu beobachten. Zu römischen Frauen als Gastgeberinnen s. Donahue 2004, a.a.O. (Anm. 3), 107-113. Für einen allgemeinen Überblick zu Frauen und Kommensalität vgl. J. Burton, 'Women's commensality in the ancient Greek world', Greece \& Rome 45 (1998), 143-165; J.-M. Pailler, 'Une place pour elle à table: le cas de Rome', Clio (Histoire, Femmes et Sociétés) 14 (2001), 119-131. 
unterscheiden: Exotisches Essen und Essenszutaten, die Verwendung von luxuriösem Geschirr, besondere Tischmanieren oder sogar eine spezielle räumliche Rahmung der Bewirtung. ${ }^{53}$ Sie dienten nach innen als Identifizierungs- und nach außen als Abgrenzungssymbole. ${ }^{54}$ Die Einladung der Magistrate zu einem gemeinsamen Essen unterschied sich von allen anderen Bewirtungen des Epameinondas durch die Auswahl des Raumes (das Heiligtum des Apollon), die Art des Mahles (auf triclinia), die Verteilung von Geld (und einer zusätzlichen Geldsumme für die Nachspeise) sowie durch den Ausschank von ,altem Wein“. Durch diese distinktiven Zeichen konstruierte sich die partikulare gesellschaftliche Identität einer Gruppe, ja ihre gesellschaftliche Position bekam dadurch einen symbolischen und systematischen Ausdruck. ${ }^{55}$ Diese im Fall des Epameinondas und der Magistrate auch visuell abgehobene Gruppe schloss sich anderen Gruppen gegenüber ab, so dass die Distanz zu anderen sozialen Gruppierungen als unüberbrückbar erscheinen mochte ${ }^{56}$ Hinzu kam der für die Versammlung dieser Gruppe gewählte Raum - „oben“ im Heiligtum -, der für eine weitere Abschottung der Mahlgemeinschaft und ihrer Tätigkeiten sorgte. ${ }^{57}$

Distinktionszeichen haben aber noch eine weitere Funktion, indem sie den sozialen Antagonismus zwischen den Mitgliedern eines solchen exklusiven Kreises in feste Bahnen lenken. So zeugte im Falle des Epameinondas das Anbieten von ,altem Wein“ statt des üblichen vom eklektischen Geschmack des Gastgebers - damit übertraf er alle seine Standesgenossen und forderte sie zur Nachahmung auf.

${ }^{53}$ Darauf hat vor allem P. Bourdieu, Die feinen Unterschiede: Kritik der gesellschaftlichen Urteilskraft (Frankfurt a.M. 1982) aufmerksam gemacht.

${ }^{54} \mathrm{Vgl}$. Grignon 2001, a.a.O. (Anm. 32), 28 f. (,segregative commensality“); Donahue 2003, a.a.O. (Anm. 32), 432-444.

${ }_{55}^{5} \mathrm{Vgl}$. Bourdieu 1982, a.a.O. (Anm. 53), 282-285.

${ }^{56}$ Vgl. Grignon 2001, a.a.O. (Anm. 32), 29, der auf das indische Kastensystem als das erfolgreichste Beispiel einer exklusiven Mahlgemeinschaft hinweist.

${ }^{57}$ Zugleich kam diese Abschottung einer öffentlichen Deklaration des exklusiven Charakters der Gruppe gleich, was M.H. Jameson, 'The spectacular and the obscure in Athenian religion', in: S. Goldhill - R. Osborne (Hrsg.), Performance Culture and Athenian Democracy (Cambridge 1999), 321-340 als "public secrecy“ bezeichnet und darin eine Strategie zur sozialen Formierung erkennt. 


\section{Die interpretatio Graeca von öffentlichen Bewirtungen nach römischem Modell}

Die Analyse des Ehrendekrets für den Euergeten Epameinondas aus Akraiphia diente dem besseren Verständnis der politischen Wirkung von öffentlichen Bewirtungsritualen. Man kann dabei beobachten, wie engagierte Gastfreundschaft bei der Veranstaltung von Festen dazu benutzt wurde, um symbolisches Kapital zu erwerben, und wie dieses wiederum in ein institutionalisiertes Bewirtungsmodell zwischen Patron und Klienten transformiert wurde. Der Einsatz eines exklusiven Bewirtungsmodus trug zudem zur Bildung eines nach außen hin stark abgegrenzten, elitären Personkreises bei und hob diesen deutlich von allen anderen gesellschaftlichen Gruppierungen ab. Mit anderen Worten: Bewirtungen boten sich als ein wichtiges agonistisches Feld an und zugleich als ein hervorragendes Instrument der sozialen Einflussnahme, dessen sich Individuen und bestimmte Gruppen bedienten, um ihre ökonomischen und politischen Ziele zu verfolgen und ihren Einfluss innerhalb der jeweiligen Gesellschaft geltend zu machen.

Aus dieser Perspektive ist die zunehmende Bedeutung von Festen und Bewirtungen während der römischen Kaiserzeit weder als Versorgungsmaßnahme für das mittelose Volk zu interpretieren ${ }^{58}$ noch darauf zu reduzieren, sie seien das am einfachsten zu handhabende Kommunikationsmedium zwischen Notabeln und dem Rest der Bevölkerung gewesen. Die Übernahme der Organisation von bereits bestehenden städtischen Festen und Opfern durch großzügige Euergeten wie Epameinondas sowie die Einführung von neuen Opfern und Bewirtungen anlässlich ihrer Amtseinsetzung als eponyme Magistrate, Gymnasiarchen oder Agonotheten bot diesem Personenkreis die Möglichkeit, ${ }^{59}$ ein bestimmtes Modell von der Bürgergemeinde in symbolischer Weise vorzustellen: Es ist das Bild einer streng hierarchisch gegliederten Gemeinschaft, innerhalb derer sich der Kreis der Notabeln nach Belieben positionieren kann. ${ }^{60}$ Im Vergleich zu dem Euergeten der hellenistischen Zeit, der sich als Indivi-

\footnotetext{
${ }^{58}$ Veyne 1976, a.a.O. (Anm. 22), 262, 283-291, 296.

${ }^{59}$ Zur Veranstaltung von öffentlichen Bewirtungen anlässlich eines Amtsantritts vgl. die Belege bei Schmitt Pantel 1981, a.a.O. (Anm. 9), 89-91; ead. 1992, a.a.O. (Anm. 2), 372-375.

${ }^{60}$ Vgl. Schmitt Pantel 1981, a.a.O. (Anm. 9), 91 f., 93.
} 
duum, als besonders vorzeigbarer Bürger präsentiert hatte, traten die Euergeten ab der späthellenistischen Zeit zugleich als Einzelpersonen und als Mitglieder einer bestimmten Gruppe auf, die sie unterstützten und mit deren anderen Mitgliedern sie gleichzeitig konkurrierten. ${ }^{61}$ Die Bezeichnung etwa ,als erster von allen“ bedeutet somit

${ }^{61}$ Die Bewirtungsinschriften von Panamara mit den verschiedenen zusätzlichen Leistungen den Gästen gegenüber sind in dieser Hinsicht sehr bezeichnend:

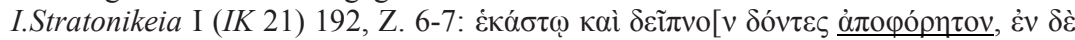

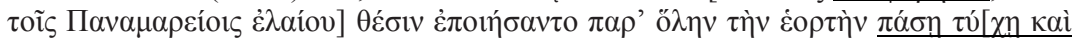

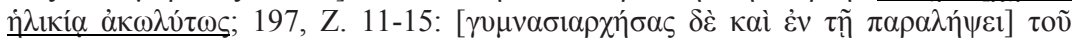

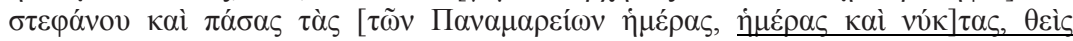

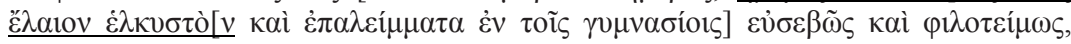

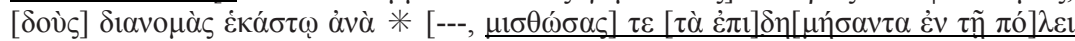

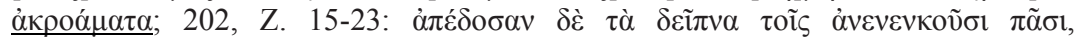

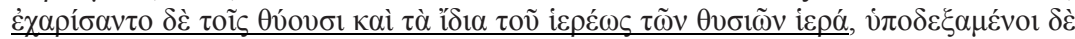

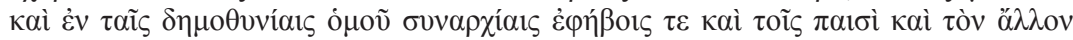

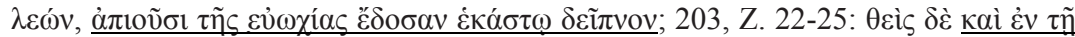

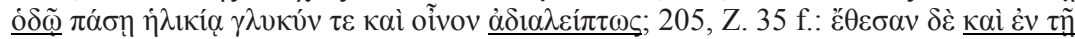

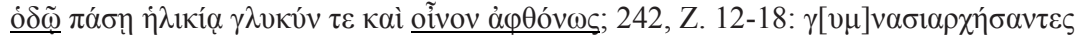

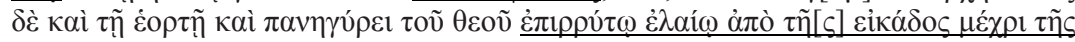

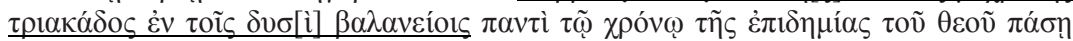

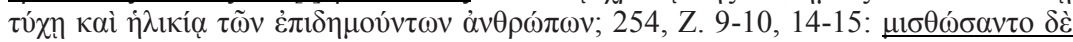

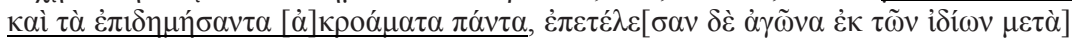

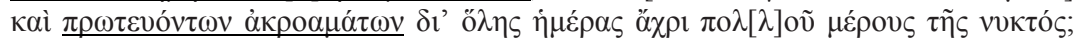

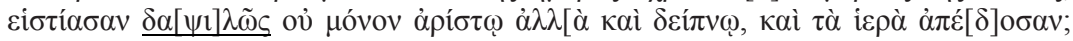

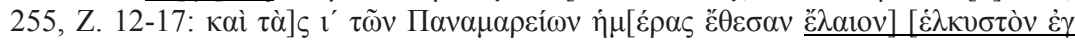

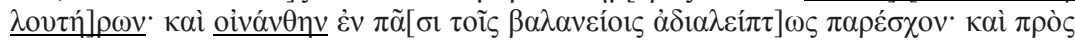

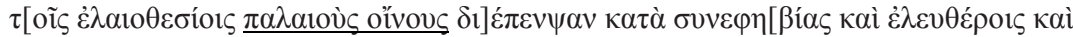

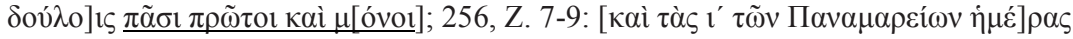

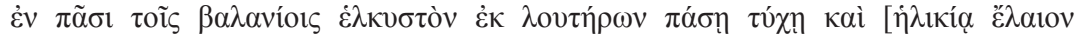

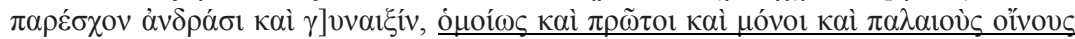

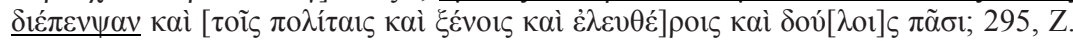

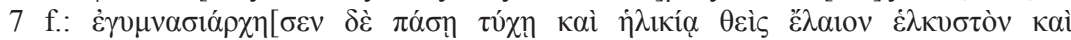

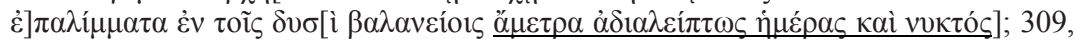

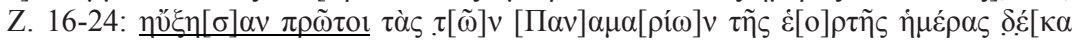

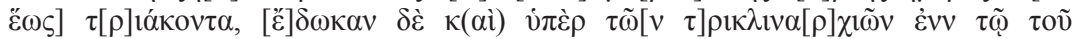

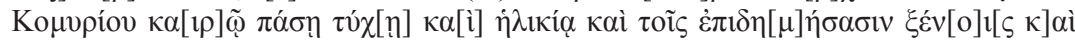

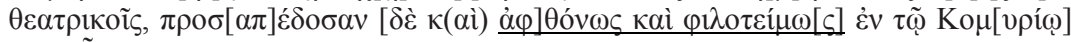

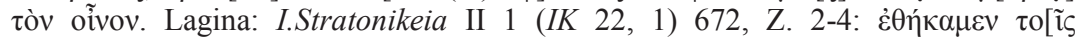

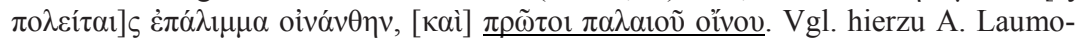
nier, Les cultes indigènes en Carie (Paris 1958), 392-406; Schmitt Pantel 1981, a.a.O. (Anm. 9), 88; ead. 1992, a.a.O. (Anm. 2), 362 f. Ein weiteres schönes Beispiel für die Konkurrenzsituation innerhalb der lokalen Eliten und die Funktion der öffentlichen Bewirtungen in diesem Rahmen stellt der Fall des dreimaligen ephesischen Asiarchen Ti. Claudius Ariston dar, der in der Regierungszeit Trajans der Beeinträchtigung der öffentlichen Ordnung beschuldigt wurde, als es bei Speisungen oder Verteilungen des Aristion zu größeren Versammlungen kam; s. Plinius Minor, 
nicht bloß ,als erster von allen Bürgern“, sondern im lokalen und überregionalen Kontext „als erster von allen Elitemitgliedern“. ${ }^{62}$ Die Bemühungen der Euergeten, sich gegenseitig zu übertreffen, führen zu einem Auswahlverfahren zwischen den Elitemitgliedern und zu einer Verfestigung sowie zu einer deutlichen Absetzung ihres Standes.

Gerade das aber war ein Novum, denn der neue Bewirtungsmodus in den griechischen Poleis nach Gruppen und nach Rang (oder sollte man lieber kata taxis sagen) scheint ein Abbild der römischen Kommensalität gewesen zu sein. Die von Epameinondas gebotenen Bewirtungen finden ohne weiteres ihr Vorbild in den cenae publicae des Augustus oder des Tiberius. Die Beschreibung der Volksbewirtung, die Tiberius im Jahre 9 v. Chr. anlässlich seines Sieges über die Pannonier veranstaltete, könnte das Skript dafür geliefert haben: Tiberius bewirtete laut Cassius Dio (55.2.4) das gesamte Volk, teils auf dem Capitol (hier feierten die Senatoren), teils auf vielen anderen Plätzen der Stadt. Livia und Iulia waren dabei die Gastgeberinnen für die Frauen. Man könnte weitere Realia anführen wie etwa die Entsprechungen zwischen dem Hapax kophinon seitou und den entsprechenden Begriffen sportula oder panaria, die die Patrone an ihre Klienten verteilten, oder zwischen der kreadosia und der visceratio; oder auf die später belegte Ablösung der Essenspakete durch Geldzahlungen - nach sozialen Gruppen geordnet - hinweisen. ${ }^{63}$ Ist also in der Umgestaltung der öffentlichen Bewirtungen ein weiterer Apekt des komplexen Prozesses auszumachen, den wir als ,Romanisierung' bezeichnen, wie vor kurzem John DONAHUE behauptet hat? $?^{64}$

Epistulae 6.31.3: dixit causam Claudius Aristion, princeps Ephesiorum, homo munificus et innoxie popularis; vgl. A.N. Sherwin-White, The Letters of Pliny: A Historical and Social Commentary (Oxford 1985, 2. Aufl.), 392 f.; weitere Beispiele für innerstädtische Rivalitäten bei Quaß 1993, a.a.O. (Anm. 10), 157-164. Allgemein zu den Motiven der Konkurrenz innerhalb der Oberschicht einer Stadt vgl. M. Sartre, L'Orient romain Provinces et sociétés provinciales en Méditerranée orientale d'Auguste aux Sévères (Paris 1991), 159-166.

${ }_{62}^{6}$ Vgl. Alföldy 1980, a.a.O. (Anm. 33), 17, 34-36.

${ }^{63} \mathrm{Zu}$ den verschiedenen Verteilungen während und im Rahmen von Bewirtungen siehe Schmitt Pantel 1992, a.a.O. (Anm. 2), 348-355 mit den Belegen; für den westlichen Teil des römischen Reiches s. Donahue 2004, a.a.O. (Anm. 3), 118-145.

${ }^{64}$ Donahue 2004, a.a.O. (Anm. 3), 4; vgl. hierzu auch die Bemerkungen von R. Gordon, 'The veil of power: emperors, sacrificers and benefactors', in: M. Beard - J. North (Hrsg.), Pagan Priests: Religion and Power in the Ancient World (London 
Das Beispiel des Epameinondas zeigt, dass die Adaption des römischen Modells einer sozial differenzierten und hierarchisierten Bewirtung der Bevölkerung durchaus den Idealen der Eliten im östlichen Teil des römischen Reiches entsprach. Die öffentlichen Bewirtungen - in ihrer nunmehr transformierten Form - eröffneten den Oberschichtangehörigen eine zusätzliche politische Arena in Bezug auf ihre Stellung innerhalb der eigenen Polis, innerhalb der eigenen Gesellschaftsschicht sowie innerhalb des römischen Reiches. Dabei stützten sie sich auf innerstädtische Entwicklungen, die in die Richtung einer markanteren sozialen Hierarchiesierung führten und wohl bereits im 2. Jh. v. Chr. begonnen hatten. Für die Römer war die unaufhörliche Versessenheit der Provinzialen auf Bewirtungen, oder sagen wir besser auf die interpretatio Graeca derselben, nur noch ein Ärgernis, wie Plinius Kaiser Trajan gegenüber zugab: Qui virilem togam sumunt vel nuptias faciunt vel ineunt magistratum vel opus publicum dedicant, solent totam bulen atque etiam e plebe non exiguum numerum vocare binosque denarios vel singulos dare (Plinius Minor, Epistulae 10.116.1).

Heidelberg, Juni 2008

1990), 229; O.M. van Nijf, The Civic World of Professional Associations in the Roman East (Amsterdam 1997), 149-152; allgemein R. MacMullen, Romanization in the Time of Augustus (New Haven 2000). 


\begin{tabular}{|c|c|c|c|c|}
\hline 竧 & 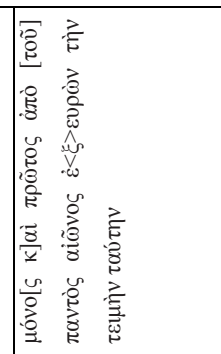 & 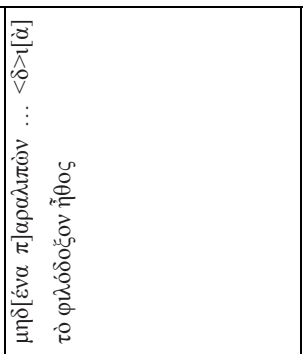 & 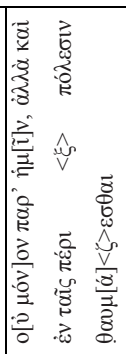 & \\
\hline 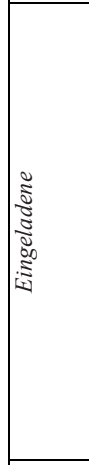 & & 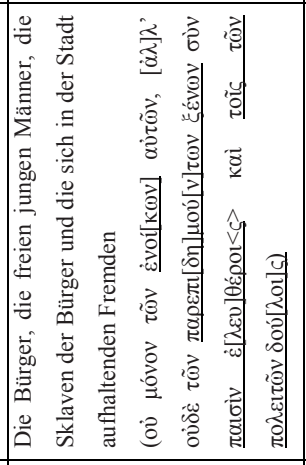 & 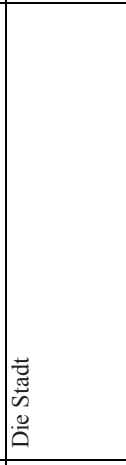 & \\
\hline 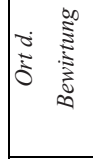 & & 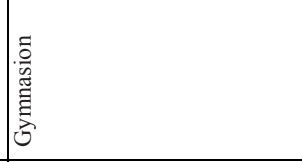 & 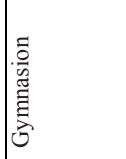 & \\
\hline 焉 & & 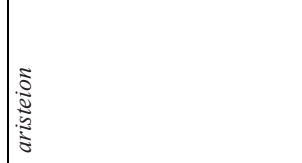 & 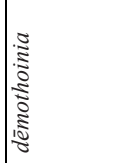 & \\
\hline 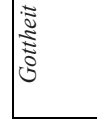 & & 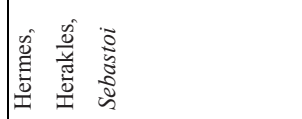 & 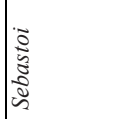 & \\
\hline 离 & 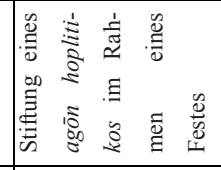 & 产 & 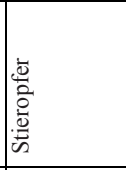 & \\
\hline है & 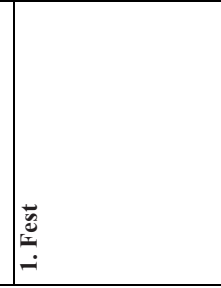 & & 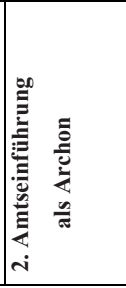 & 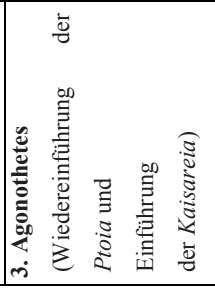 \\
\hline
\end{tabular}




\begin{tabular}{|c|c|c|c|c|c|c|c|}
\hline & 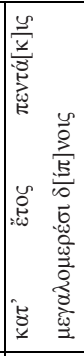 & 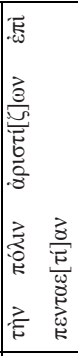 & 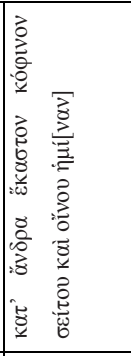 & 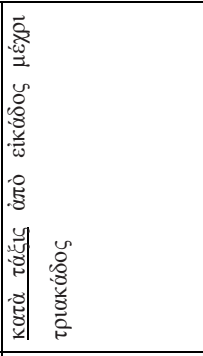 & 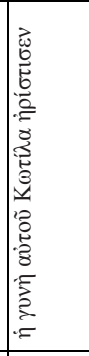 & 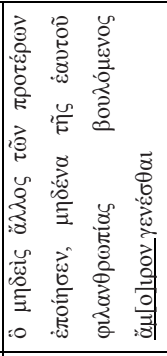 & 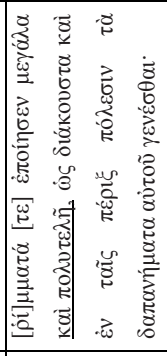 \\
\hline & 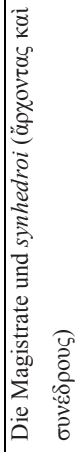 & 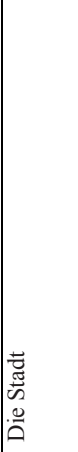 & 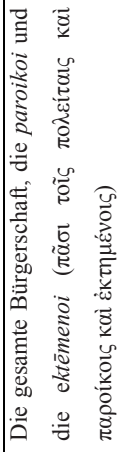 & 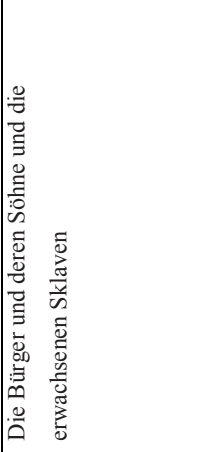 & 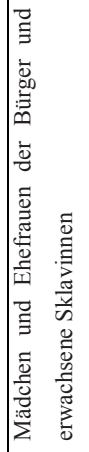 & 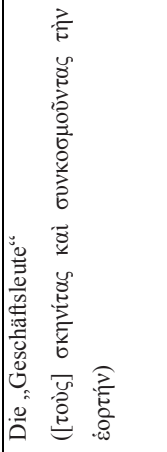 & 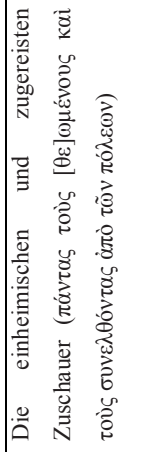 \\
\hline & & & & & & 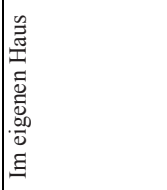 & 离 \\
\hline & 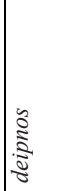 & 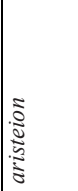 & 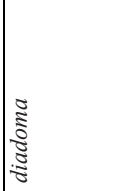 & 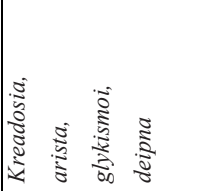 & 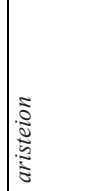 & 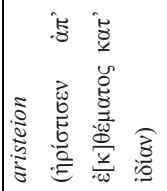 & $\mid$ \\
\hline & & & & 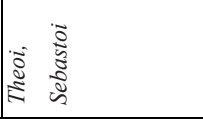 & & & \\
\hline 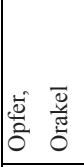 & & & 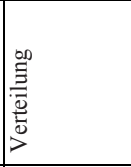 & 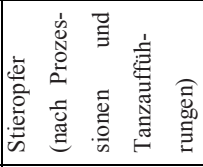 & & & \\
\hline 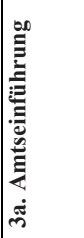 & & & ले & & & & \\
\hline
\end{tabular}




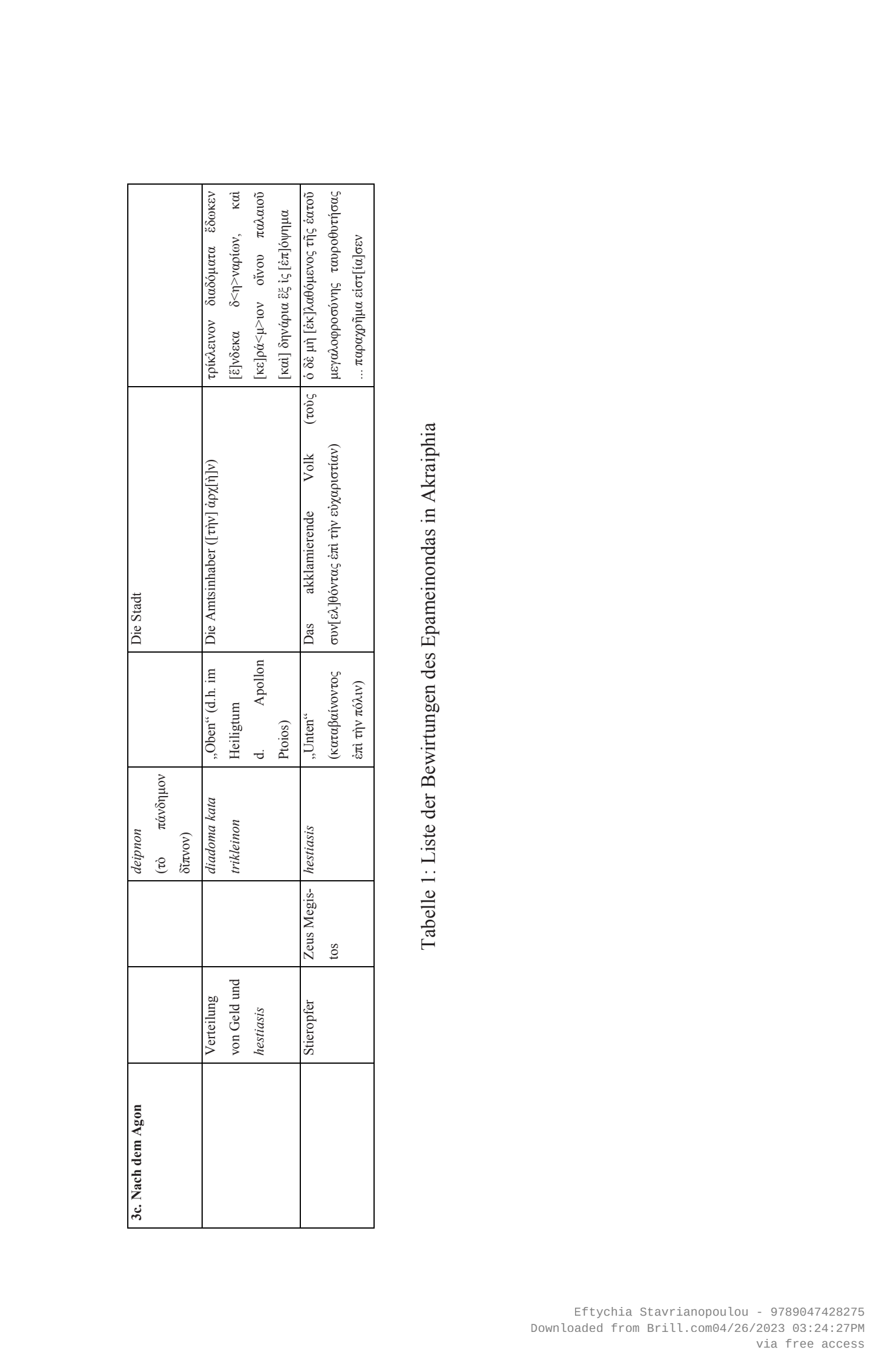

